\title{
Online Estimation of Wind Turbine Tip Speed Ratio by Adaptive Neuro-Fuzzy Algorithm
}

\author{
Aamer Bilal Asghar ${ }^{1}$, Xiaodong Liu $^{2}$ \\ School of Control Science \& Engineering \\ Faculty of Electronic Information \& Electrical Engineering \\ Dalian University of Technology \\ Dalian 116024, P.R. China
}

\begin{abstract}
The efficiency of a wind turbine highly depends on the value of tip speed ratio during its operation. The power coefficient of a wind turbine varies with tip speed ratio. For maximum power extraction, it is very important to hold the tip speed ratio at optimum value and operate the variable-speed wind turbine at its maximum power coefficient. In this paper, an intelligent learning based adaptive neuro-fuzzy inference system (ANFIS) is proposed for online estimation of tip speed ratio (TSR) as a function of wind speed and rotor speed. The system is developed by assigning fuzzy membership functions (MFs) to the input-output variables and artificial neural network (ANN) is applied to train the system using back propagation gradient descent algorithm and least square method. During the training process, the ANN adjusts the shape of MFs by analyzing training data set and automatically generates the decision making fuzzy rules. The simulations are done in MATLAB for standard offshore $5 \mathrm{MW}$ baseline wind turbine developed by national renewable energy laboratory (NREL). The performance of proposed neuro-fuzzy algorithm is compared with conventional multilayer perceptron feed-forward neural network (MLPFFNN). The results show the effectiveness of proposed model. The proposed system is more reliable for accurate estimation of tip speed ratio.
\end{abstract}

Keywords-Wind speed; rotor speed; power coefficient; tip speed ratio; ANFIS

\section{INTRODUCTION AND MOTIVATION}

Wind is a sustainable source of energy and is considered as a best alternate of limited fossil fuel resources. Wind power is economical and environment friendly. Wind power generation system mainly consists of a wind turbine and an electric generator. Wind turbine blades convert the kinetic energy of wind into mechanical energy and generator converts the mechanical energy into electrical energy. Wind turbine plays a vital role in power generation. The wind turbine rotor speed must be controlled in varying wind speed conditions [1].

The mechanical power captured by wind turbine depends on the value of power coefficient $\left(C_{P}\right)$. Wind turbine operating at maximum value of power coefficient $\left(C_{P \max }\right)$ captures maximum power from wind. The power coefficient is a nonlinear function of tip speed ratio $(\lambda)$ and blade pitch angle $(\beta)$. Wind turbine achieves maximum value of power coefficient ( $C_{P \max }$ ) at optimal tip speed ratio $\left(\lambda_{\text {opt }}\right)[2],[3]$.
TSR actually compares the rotor speed with the wind speed ( $v$ ). Higher rotor speed as compared to wind speed means higher TSR. The wind turbine has maximum efficiency at optimal TSR $\left(\lambda_{\text {opt }}\right)$ which corresponds to maximum power coefficient ( $C_{P \max }$ ). Therefore, it is very important to control the rotor speed according to the available wind speed. The optimal tip speed ratio $\left(\lambda_{\text {opt }}\right)$ depends on many factors such as number of blades, size (length) of blade and the shape of airfoil used [4]. The lesser number of blades and longer blade length leads to higher optimal TSR. Small wind turbines have higher rotational speed but as their blade length is small, so their optimal TSR is not as much as large wind turbines. The optimal TSR for single blade and 2 blade wind turbine is around 9 and 6 respectively. The optimal TSR for a 3 blade wind turbine is 5 which can be further improved up to 7 or 8 by using highly efficient and well-designed airfoil rotor blades. Generally, it is taken as 7 for a 3 blade wind turbine. The optimal TSR for a 4 blade wind turbine is around 3 . The flow of air around wind turbine blades produces aerodynamic noise which keeps growing with wind speed. The speed of blade tip (end point of the blade) is a critical factor in deciding the aerodynamic noise produced by the wind turbine. Wind turbine with higher TSR produces more aerodynamic noise [5]. Moreover, the aerodynamic noise also depends on the shape of blade. Thin airfoil is used because of its aerodynamic benefits [6], [7]. A 3 blade wind turbine produces less noise as compared to a 2 blade wind turbine of the same size because of its lesser TSR. The higher number of blades produces more torque. The wind turbines having more than three blades are used for water pumping and grinding purposes. But, 4 blade wind turbines are not used for power generation because of their much higher cost, weight and less profit in terms of efficiency gain $(0.5 \%)$ or power capturing. On the other hand, 2 blade wind turbines are also not preferred for power generation due to their higher aerodynamic noise. Moreover, 2 blade wind turbines with same size have higher TSR and in turn higher rotor speed, which increases the apparent weight (higher structural loading) of the blades. Therefore, brakes can fail in strong wind and a 2 blade wind turbine can collapse. So, a 3 blade design can produce more power at slower rotational speed than a 2 blade design and more cost efficient as compared to a 4 blade option. Therefore, 3 blade wind turbines are mostly used for power generation. 
TSR varies with wind speed and tip speed of wind turbine. TSR should be maintained around its optimal value to extract maximum power. It is very important to estimate the TSR of wind turbine to control the power produced during its operation. In [8], [9] ANN based structure was built to predict the tip speed ratio for wind turbine profile types NACA 4415 and LS-1. The system was capable to efficiently estimate the value of TSR. An adaptive perturbation and observation $(\mathrm{P} \& \mathrm{O})$ method was proposed in [10] for estimation of TSR and maximum power point tracking (MPPT). The proposed system was found more efficient than the classical perturbation and observation method. In [11], [12] tip speed ratio and MPPT was performed by measuring the wind speed and generator voltage and current. The designed system was implemented on a small scale wind turbine to validate its performance. In [13] two ANN models were proposed, one to operate the variable-speed wind turbine at optimum TSR for MPPT and the second ANN model for pitch control. The proposed model was found to have the capability of MPPT for varying wind speed.

The perturbation and observation $(\mathrm{P} \& \mathrm{O})$ based algorithm is one of the most widely used methods for MPPT of wind turbine. The main disadvantage associated to this technique is that it keeps on oscillating around the maximum power point (MPP). Therefore, it does not exactly track the MPP which results in losing some part of the available power. However, this discrepancy can be covered by using smaller perturbation step. But it makes the algorithm slow in response and also affects the performance of controller to optimize the dynamic response of system. So, we have to compromise between reduced oscillations and fast dynamic response. The adaptive $\mathrm{P} \& \mathrm{O}$ method involves complex computation of derivatives. So, there is need to use some soft computing based techniques. The ANN is the most widely used soft computing technique to estimate the TSR. The ANNs have shown much better performance over conventional methods. The accuracy of ANN depends on the number of hidden layer neurons and the type of activation function used. The learning rate and optimization methods used (back propagation algorithm or Levenberg-Marquardt algorithm) also effect the performance. The training of ANN may be time taking as it involves many tuning parameters.

Hybrid intelligent systems are becoming very popular because of their ability to dealing with nonlinear systems. Hybrid systems are actually the combination of two different techniques. These systems are capable of realizing the nonlinear relationships with much higher accuracy. The ANFIS is also a type of hybrid systems. ANFIS is a combination of Takagi-Sugeno fuzzy inference system (T-S FIS) and ANN. Fuzzy systems make decisions of the basis of human reasoning and ANNs are trained to learn from the previous experiences or data. Researchers have successfully implemented ANFIS to deal fitting, forecasting, regression and classification problems [14]-[16].

The major aim of this study is to propose a hybrid intelligent learning based neuro-fuzzy methodology for accurate estimation of TSR for offshore $5 \mathrm{MW}$ baseline wind turbine. The experimental data of wind turbine is collated to design the ANFIS model. The ANN trains the system using hybrid learning method. The results of ANFIS are then compared with conventional MLPFFNN. The results show that ANFIS can estimate the TSR with much higher accuracy as compared to MLPFFNN.

\section{WIND POWER FUNDAMENTALS AND SPECIFICATIONS OF NREL OFFSHORE 5 MW BASELINE WIND TURBINE}

The mechanical power ' $P_{m}$ ' captured by a wind turbine is expressed as,

$$
P_{m}=\frac{1}{2} \rho A v^{3} C_{P}(\lambda, \beta)
$$

Here, ' $\rho$ ' is the density of air in $\left(\mathrm{kg} / \mathrm{m}^{3}\right)$, ' $A$ ' is the area of the wind turbine rotor in $\left(\mathrm{m}^{2}\right)$ and ' $v$ ' is the wind velocity in $(\mathrm{m} / \mathrm{s})$. The wind turbine power coefficient ' $C_{P}$ ' is the measure of the efficiency of wind turbine. The power coefficient varies with the TSR $(\lambda)$ and blade pitch angle $(\beta)$.

The NREL virtually designed an offshore $5 \mathrm{MW}$ baseline wind turbine to standardize the advancements is new techniques [17]. Researchers around the world use this model as a reference to investigate the aerodynamics and control of wind turbine. The specifications of offshore $5 \mathrm{MW}$ baseline wind turbine are given in Table I.

As mentioned in Table I, the wind turbine reaches to its maximum power coefficient of 0.4868 at TSR of 7.55. The $C_{P}$-TSR curve of $5 \mathrm{MW}$ wind turbine is shown in Fig. 1.

TABLE I. SPECIFICATIONS OF NREL OFFSHORE 5 MW BASELINE WIND TURBINE

\begin{tabular}{|l|l|}
\hline Parameter & Value \\
\hline Rated power & $5 \mathrm{MW}$ \\
\hline Rotor configuration & Upwind,3 blades \\
\hline Rotor, hub diameter & $126 \mathrm{~m}, 3 \mathrm{~m}$ \\
\hline Hub height & $90 \mathrm{~m}$ \\
\hline Cut-in, rated, cut-out wind speed & $3 \mathrm{~m} / \mathrm{s}, 11 \mathrm{~m} / \mathrm{s}, 25 \mathrm{~m} / \mathrm{s}$ \\
\hline Cut-in, rated rotor speed & $6.9 \mathrm{rpm}, 12.1 \mathrm{rpm}$ \\
\hline Rated generator speed & $1174 \mathrm{rpm}$ \\
\hline Peak power coefficient & 0.4868 \\
\hline Tip speed ratio at peak power coefficient & 7.55 \\
\hline $\begin{array}{l}\text { Collective blade pitch angle at peak power } \\
\text { coefficient }\end{array}$ & $0^{\circ}$ \\
\hline
\end{tabular}

When ' $\lambda<\lambda_{\text {opt }}$ ', the turbine speed is slow and a large fraction of undisturbed wind passes through the blades. Therefore, less energy is harnessed because of poorly designed rotor blades. When ' $\lambda=\lambda_{\text {opt }}$ ', the wind turbine operates at maximum value of power coefficient $\left(C_{P \max }\right)$. Therefore, maximum energy is harnessed. When ' $\lambda>\lambda_{\text {opt }}$ ', 
the turbine speed is so fast that the rotor behaves like a disk (wall). Therefore, less energy is harnessed. The wind turbine rotor is highly stressed and there is a risk of structure failure.

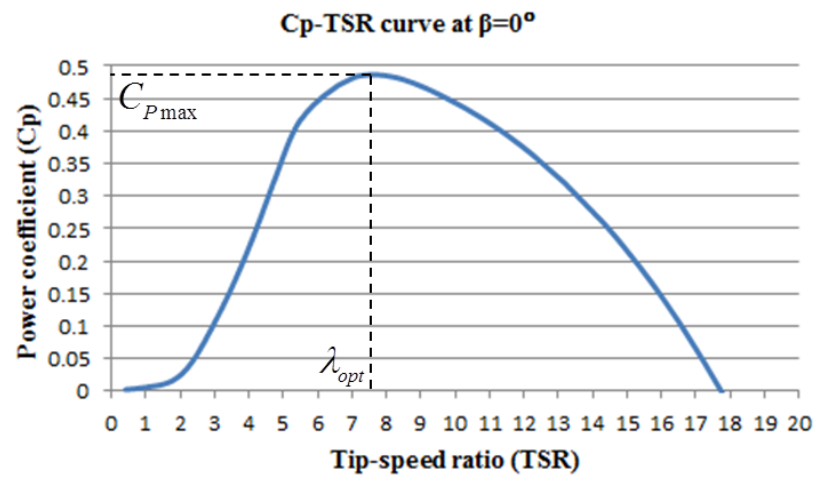

Fig. 1. $C_{P}$-TSR curve for NREL offshore $5 \mathrm{MW}$ baseline wind turbine at pitch angle $(\beta)$ of $0^{\circ}$.

The statistical properties of experimental data collected from offshore $5 \mathrm{MW}$ baseline wind turbine are shown in Table II. The total number of collected data samples is 338 . The collected data set is subdivided into training data and testing data. The training data is used to train the ANFIS based TSR estimator and then its performance is checked for testing data.

TABLE II. Statistical Properties of Data used to Design ANFIS BASED TSR ESTIMATOR

\begin{tabular}{|l|l|l|l|}
\hline Wind turbine parameters & Average value & Max. value & Min. value \\
\hline TSR & 5.41 & 32.96 & 0.37 \\
\hline Rotor speed (rad/s) & 0.8529 & 1.5707 & 0.1047 \\
\hline Wind speed (m/s) & 13.834 & 25 & 3 \\
\hline
\end{tabular}

III. METHODOLOGY

\section{A. Structure of Adaptive Neuro-Fuzzy Inference System (ANFIS)}

ANFIS is a soft computing technique to deal with highly nonlinear systems. It combines the features of T-S FIS and ANN in a single frame work to provide accurate decision making results. The ANFIS is a computationally intelligent system which involves parallel computing. The ANN uses the training data to update the parameters of fuzzy MFs [18]. The basic structure of ANFIS is shown in Fig. 2.

The output of first order T-S FIS with two IF-THEN fuzzy rules can be expressed as,

Rule-1: If ' $x$, is $A_{1}$ and ' $y$, is $B_{1}$ then $f_{1}=p_{1} x+q_{1} y+r_{1}$

Rule-2: If ' $x$, is $A_{2}$ and ' $y$ ' is $B_{2}$ then $f_{2}=p_{2} x+q_{2} y+r_{2}$

Here, ' $x$ ' and ' $y$ ' are two inputs and ' $f$ ' is the output.
Where, ' $p$ ', ' $q$ ' and ' $r$ ' are consequent parameters of the first order polynomial.

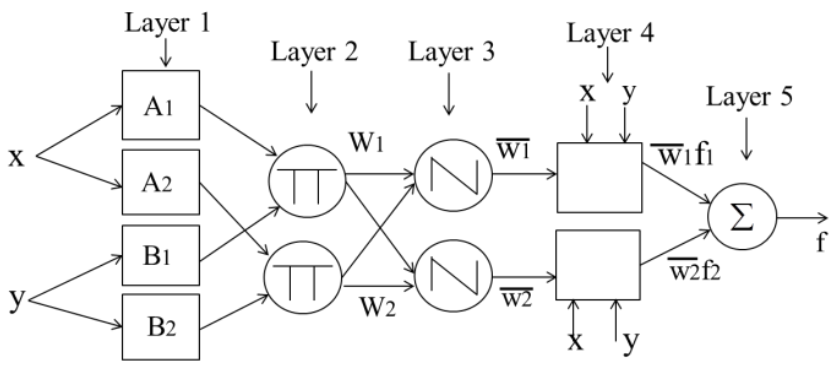

Fig. 2. The basic architecture of ANFIS.

ANFIS architecture consists of five layers of neurons. The first layer is called fuzzification layer and each neuron in this layer is adaptive. In first layer, MFs are assigned to each input variable. The input MFs can be bell-shaped, Gaussian, triangular, trapezoidal and dsigmoid, etc.

Each neuron in second layer computes the firing strength of fuzzy rule by multiplying the incoming signals.

$$
W_{i}=\mu_{A i}(x) \cdot \mu_{B i}(y)
$$

The neurons in third layer calculate the normalized firing strength of each rule.

$$
\overline{W_{i}}=\frac{W_{i}}{\sum W_{i}}
$$

Each neuron in the fourth layer is adaptive and this layer is called defuzzification layer. Each neuron multiplies the normalized firing strength of a rule with corresponding output polynomial function to get a crisp output.

$$
\bar{W}_{i} f_{i}=\bar{W}_{i}\left(p_{i} x+q_{i} y+r_{i}\right)
$$

The fifth layer has a single neuron which sums up all incoming signals from layer 4 and produces a single crisp output.

$$
f=\sum \overline{W_{i}} \cdot f_{i}=\frac{\sum W_{i} \cdot f_{i}}{\sum W_{i}}
$$

\section{DEVELOPING THE TSR ESTIMATORS}

\section{A. ANFIS based Estimator}

The ANFIS analyzes the input-output training data set and trains the system using hybrid learning algorithm. Experiments are conducted for each type of input MF and ANFIS is designed using grid partitioning. Six MFs are assigned to each input parameters (wind speed and rotor speed) and linear MFs to output (TSR). The system is trained for 200 epochs and error tolerance is set to zero. After the completion of training process, ANFIS automatically generated 36 IF-THEN fuzzy rules for input-output mapping. The training and testing errors produced by ANFIS using different type of input MFs are given in Table III. It has been 
noticed that bell-shaped MFs provide least training and testing error followed by Gaussian, dsigmoid, triangular and trapezoidal MFs.

Mathematically, the bell-shaped MF is expressed as,

$$
\mu_{A i}(x)=\frac{1}{1+\left|\frac{x-c_{i}}{a_{i}}\right|^{2 b_{i}}}
$$

Where, ' $a$ ', ' $b$ ' and ' $c$ ' are premise parameters and ' $i$ ' is equal to total number of MFs assigned to each input parameter.

During the learning process, the consequent parameters of polynomial functions are updated in the forward pass using least square algorithm and premise parameters of bell-shaped MFs are updated in backward pass using backpropagation gradient descent algorithm. The trained bell-shaped input MFs are shown in Fig. 3 and 4. The decrease in root mean square error (RMSE) during the training of bell-shaped MFs for 200 epochs is shown in Fig. 5.

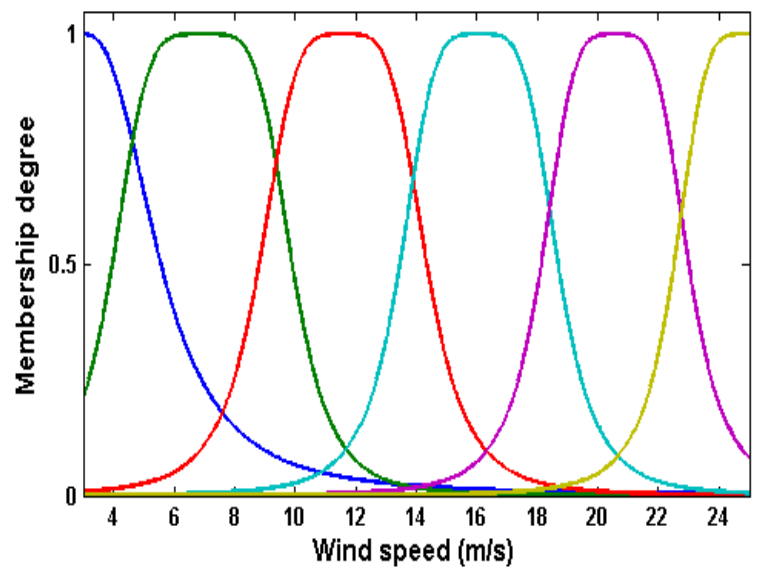

Fig. 3. Trained bell-shaped MFs of wind speed.

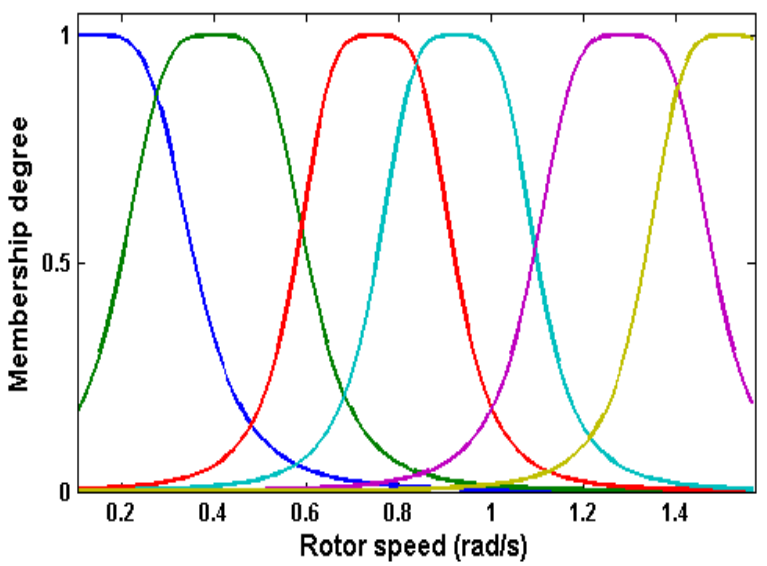

Fig. 4. Trained bell-shaped MFs of rotor speed.

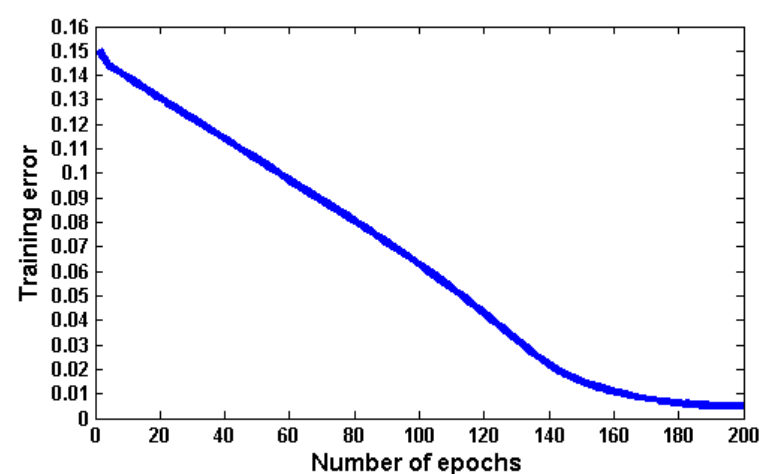

Fig. 5. Training of bell-shaped MFs for 200 epochs.

TABLE III. TRAINING AND TESTING ERRORS PRODUCED BY TSR ESTIMATOR FOR DIFFERENT TYPE OF INPUT MFS

\begin{tabular}{|l|l|l|l|l|l|}
\hline $\begin{array}{c}\text { Type of input } \\
\text { MFs }\end{array}$ & $\begin{array}{c}\text { No. of } \\
\text { input } \\
\text { MFs }\end{array}$ & $\begin{array}{c}\text { Type of } \\
\text { output } \\
\text { MFs }\end{array}$ & $\begin{array}{c}\text { No. of } \\
\text { epochs }\end{array}$ & $\begin{array}{c}\text { Training } \\
\text { error }\end{array}$ & $\begin{array}{c}\text { Testing } \\
\text { error }\end{array}$ \\
\hline Bell-shaped & 6 & Linear & 200 & 0.005299 & 0.014567 \\
\hline Gaussian & 6 & Linear & 200 & 0.027864 & 0.034992 \\
\hline dsigmoid & 6 & Linear & 200 & 0.061608 & 0.086566 \\
\hline Triangular & 6 & Linear & 200 & 0.097743 & 0.1421 \\
\hline Trapezoidal & 6 & Linear & 200 & 0.11339 & 0.12391 \\
\hline
\end{tabular}

\section{B. MLPFFNN based Estimator}

MLPFFNN is a biological inspired system which consists of different layers of neurons. Each neuron is a processing unit which processes the information until a threshold is obtained and then passes on the information to the next neuron. MLPFFNN is mainly composed of three layers of neurons. First layer is called input layer, second layer is called hidden layer and third layer is called output layer. The neurons in one layer are directly connected to the neurons of next layers. The basic structure of MLPFFNN is shown in Fig. 6.

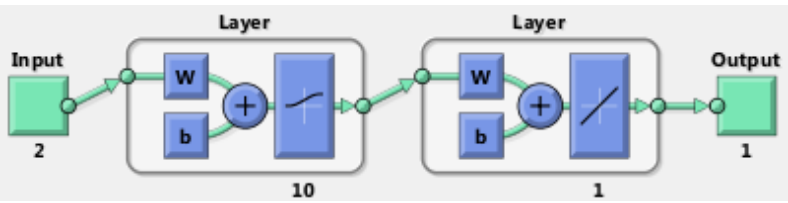

Fig. 6. Structure of designed MLPFFNN.

There are two inputs of the TSR estimator which are wind speed and rotor speed, so there are two neurons in the first layer (input layer). While designing MLPFFNN, ten neurons are assigned to the hidden layer and logistic sigmoid 'logsig' transfer function is used as activation function for hidden layer neurons. The logistic sigmoid function can be mathematically expressed as,

$$
\log \operatorname{sig}(x)=\frac{1}{1+e^{-x}}
$$

The linear transfer function is used as activation function for output layer neuron. In order to train the system, the learning rate is selected as 0.01 and error goal as 0.0001 . The Levenberg-Marquardt back propagation algorithm is used to 
train the system for 200 epochs. The mean square error (MSE) produced by MLPFFNN after 200 epochs is 0.041279 as shown in Fig. 7.

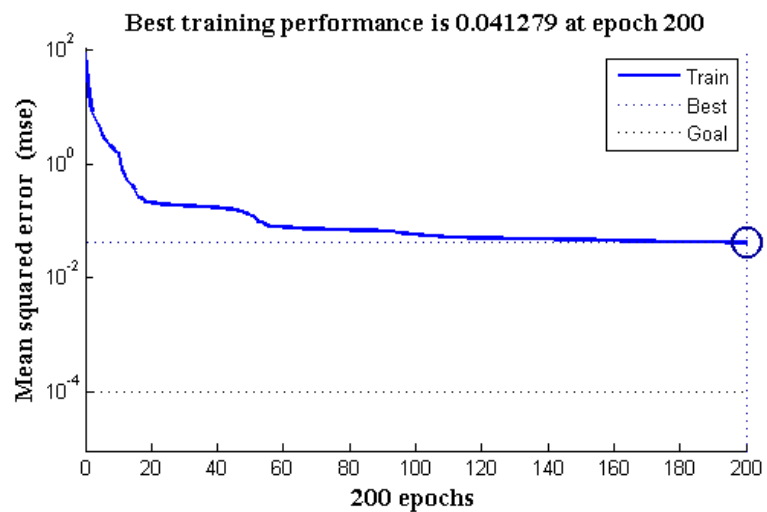

Fig. 7. The MSE produced after 200 epochs.

The MSE and RMSE produced by ANFIS and MLPFFNN are given in Table IV. It is found that ANFIS based estimator provides the best fit of the actual TSR with minimum MSE and RMSE.

TABLE IV. PERFORMANCE INDICES OF BOTH APPROACHES

\begin{tabular}{|l|l|l|}
\hline Model & MSE & RMSE \\
\hline ANFIS & 0.00002808 & 0.0052991 \\
\hline MLPFFNN & 0.041279 & 0.203172 \\
\hline
\end{tabular}

\section{CONCLUSION}

The wind turbine efficiency depends on the value of power coefficient, which varies with the TSR. Therefore, it is necessary to accurately estimate the value of TSR to produce optimum power. In this study, a hybrid intelligent methodology based of ANFIS is proposed for accurate estimation of TSR. The experimental data is collected from NREL offshore $5 \mathrm{MW}$ baseline wind turbine. The T-S FIS of ANFIS is designed by assigning bell-shaped, Gaussian, dsigmoid, triangular and trapezoidal MFs to the inputs. Then ANN is used to train the system for 200 epochs using hybrid learning method. It is noticed that ANFIS provides the best results for bell-shaped MFs. Then, conventional MLPFFNN based TSR estimator is designed for the same data. The logistic sigmoid and linear transfer functions are used as activation function for hidden layer and output layer, respectively. The system is trained for 200 epochs using Levenberg-Marquardt back propagation algorithm. The results of ANFIS and MLPFFNN are compared with reference to actual value of TSR. It has been observed that ANFIS has much better performance than conventional MLPFFNN and produces less RMSE even for any type of input MF. Therefore, ANFIS is selected as the best suitable technique for accurate estimation of TSR. The ANFIS involves fewer adjustable (tuning) parameters as compared to ANNs; therefore its training is less time consuming and easy. The ANFIS does not require any detailed and precise mathematical model of the system. It only incorporates the prior knowledge of the system to adapt the system behavior. Moreover, ANFIS does not involve complex computations therefore it is less computationally complex as compared to other conventional approaches. ANFIS automatically generates the IF-THEN fuzzy rules by analyzing the training data. ANFIS uses the minimum number of fuzzy rules which makes the decision making process fast. ANFIS is robust and adaptive to changing conditions. ANFIS is simple, cheaper to develop and can easily be implemented by using data acquisition cards.

The results presented in this paper are subject to some hardware limitations. The data set used in this study is collected from variable-speed variable-pitch $5 \mathrm{MW}$ offshore wind turbine which is virtually developed by NREL to support the conceptual study of wind turbine aerodynamics and control properties. The actual wind turbine system is not only expensive but also much larger in size. Therefore, it is not usually available in the laboratories, except those which are highly equipped and solely dedicated for wind energy research. The ideal situation is to collect the data samples from an operational wind turbine system and then using the collected data to design estimation and control mechanism.

\section{FUTURE WORK}

The optimum TSR is different for every wind turbine type. In future, the proposed approach can be implemented on any wind turbine model in similar fashion to accurately estimate the TSR after collecting the appropriate data. The estimated value of optimum TSR can also be further utilized to design MPPT controller. Future research also includes the implementation of proposed TSR estimator on real wind turbine system to verify the results.

\section{ACKNOWLEDGMENT}

This research work is funded by the Natural Science Foundation of China under grant 61673082, 61175041 and 61533005.

\section{REFERENCES}

[1] A. B. Asghar and X. Liu, "Estimation of wind turbine power coefficient by adaptive neuro-fuzzy methodology," Neurocomputing, Vol. 238, pp. 227-233, 2017.

[2] A. B. Asghar and X. Liu, "Adaptive neuro-fuzzy algorithm to estimate effective wind speed and optimal rotor speed for variable-speed wind turbine," Neurocomputing, Vol. 272, pp. 495-504, 2018.

[3] H. Yu, Y. He, Z. Zhao and M. Wu, "Research on fuzzy PID pitchcontrolled system based on SVM," J. Adv. Comput. Intell. Intell. Inform., Vol. 20, No.2, 2016.

[4] J. G. Njiri and D. Söffker, "State-of-the-art in wind turbine control: Trends and challenges," Renew. Sust. Energy Rev., Vol. 60, pp. 377393, 2016.

[5] M. H. Mohamed, "Aero-acoustics noise evaluation of H-rotor Darrieus wind turbines," Energy, Vol. 65, pp. 596-604, 2014.

[6] H. R. Kaviani and A. Nejat, "Aerodynamic noise prediction of a MWclass HAWT using shear wind profile," J. Wind Eng. Ind. Aerodyn., Vol. 168, pp. 164-176, 2017.

[7] M. H. Mohamed, "Reduction of the generated aero-acoustics noise of a vertical axis wind turbine using CFD (Computational Fluid Dynamics) techniques," Energy, Vol. 96, pp. 531-544, 2016.

[8] M. A. Yurdusev, R. Ata and N. S. Cetin, "Assessment of optimum tip speed ratio in wind turbines using artificial neural networks," Energy, Vol. 31, pp. 2153-2161, 2006.

[9] R. Ata and Y. Kocyigit, "An adaptive neuro-fuzzy inference system approach for prediction of tip speed ratio in wind turbines," Expert Syst. Appl., Vol. 37, pp. 5454-5460, 2010. 
[10] A. J. Mahdi, W. H. Tang and Q. H Wu, "Estimation of tip speed ratio using an adaptive perturbation and observation method for wind turbine generator systems," in: Proceedings of the IET Conference on Renewable Power Generation (RPG), Edinburgh, UK, pp. 1-6, 6-8 Sept. 2011.

[11] J. D. M. De Kooning, L. Gevaert, J. Van de Vyver, T.L. Vandoorn and L. Vandevelde, "Online estimation of the power coefficient versus tipspeed ratio curve of wind turbines," in: 39th annual conference of the IEEE Industrial-Electronics-Society (IECON), Vienna, Austria, pp. 1792-1797, 10-14 November 2013.

[12] L. Gevaert, J. D. M. De Kooning, T.L. Vandoorn, J. Van de Vyver and L. Vandevelde, "Evaluation of the MPPT performance in small wind turbines by estimating the tip-speed ratio," in: 48th International Universities Power Engineering Conference (UPEC), IEEE, pp. 1-5, 2-5 September 2013.

[13] H. H. El-Tamaly and A. Y. Nassef, "Tip speed ratio and pitch angle control based on ANN for putting variable speed WTG on MPP," in: 18th International middle-east power systems conference (MEPCON), IEEE Power \& Energy Soc, Helwan Univ., Cairo, Egypt, pp. 625-632, 27-29 December 2016.

[14] M. Negnevitsky, D. Nikolic and M. de Groot, "Adaptive neuro-fuzzy synchronization in isolated power systems with high wind penetration," J. Adv. Comput. Intell. Intell. Inform., Vol. 20, No.3, 2016.

[15] S. Roy, A. K. Das, V. S. Bhadouria, S. R. Mallik, R. Banerjee and P.K. Bose, "Adaptive-neuro fuzzy inference system (ANFIS) based prediction of performance and emission parameters of a CRDI assisted diesel engine under CNG dual-fuel operation," J. Nat. Gas Sci. Eng., Vol. 27, pp. 274-283, 2015.

[16] R. Dash and P.K. Dash, "Efficient stock price prediction using a selfevolving recurrent neuro-fuzzy inference system optimized through a modified differential harmony search technique," Expert syst. Appl., Vol. 52, pp. 75-90, 2016.

[17] J. Jonkman, S. Butterfield, W. Musial and G. Scott, "Definition of a 5MW reference wind turbine for offshore system development," National renewable energy laboratory, Golden, CO, USA, 2009 Technical report NREL/TP-500-38060.

[18] A. B. Asghar and X. Liu, "Estimation of wind speed probability distribution and wind energy potential using adaptive neuro-fuzzy methodology," Neurocomputing, Vol. 287, pp. 58-67, 2018. 2018.

\section{AUTHOR's PROFILE}

Aamer Bilal Asghar received the B.S. degree in Electronic Engineering from The Islamia University of Bahawalpur, Pakistan in 2008, the M.S. degree in Electrical Engineering from Government College University Lahore, Pakistan in 2014. Currently, he is a Ph.D. student in School of Control Science and Engineering at Dalian University of Technology, Dalian, P.R. China. He is serving as a Lecturer in Department of Electrical Engineering at COMSATS Institute of Information Technology, Lahore, Pakistan since 2014. His research interest areas include fuzzy control, artificial neural networks, ANFIS, genetic algorithm, granular computing, machine learning and intelligent control.

Xiaodong Liu received the B.S. degree in Mathematics from Northeast Normal University, Jilin, P.R. China in 1986, the M.S. degree in Mathematics from Jilin University, Jilin, P.R. China in 1989, and the Ph.D. degree in Information Science and Engineering from Northeastern University, Shenyang, P.R. China in 2003. Currently, he is a professor in School of Control Science and Engineering at Dalian University of Technology, Dalian, P.R. China. His research areas include fuzzy control, axiomatic fuzzy sets, machine learning, data mining, artificial intelligence, time series analysis and financial mathematics. 